\title{
Enterococcus spp. isolated from root canals with persistent chronic apical periodontitis in a Chilean population
}

\author{
Gabriela Sánchez-Sanhueza ${ }^{1}$, Gerardo González-Rocha², Mariana Dominguez², Helia Bello-Toledo²
}

${ }^{1}$ Universidad de Concepción, Faculty of Dentistry, Department of Restorative Dentistry, Concepción, Chile

Universidad de Concepción, Faculty of Biological Sciences, Department of Microbiology, Research Laboratory on Antibacterial Agents, Concepción, Chile

\begin{abstract}
Aim: To isolate and identify in a Chilean population, Enterococcus spp. from root canals with persistent chronic apical periodontitis (CAP) and to investigate the potential correlation between the bacteria and the observed clinical features. Methods: Twenty patients with indication for endodontic retreatment due to persistent CAP were selected. Data from patient general health and dental clinical history were recorded. During retreatment, a microbial sample was obtained from the root canal and inoculated in a selective Enterococcus medium. Using bacterial cultivation methods, bacterial isolates belonging to the genus Enterococcus were identified. The relationship between the number of colony-forming units of Enterococcus spp. and patient clinical data was assessed statistically by the Pearson Chi square and Fisher exact tests. Finally, a Polymerase Chain Reaction (PCR) assay to determine the most prevalent species of Enterococcus spp. was conducted in the clinical samples, and the results were analyzed by a proportion comparison test. Results: Enterococcus spp. strains were isolated in $70 \%$ of the patients. Most of them (98.8\%) accounted for Enterococcus faecalis and only 1.2\% for Enterococcus faecium. A high frequency of $E$. faecalis was found in teeth with inadequate endodontic treatment or dental crown restorations. Conclusions: This study concluded that $E$. faecalis is prevalent in root canals with persistent CAP in a Chilean population. E. faecium as found in a single case with the poorest root canal filling. Further studies are still required to investigate the presence of other species, which may be linked to persistent chronic apical periodontitis.
\end{abstract}

Keywords: periapical granuloma; Enterococcus faecalis; Enterococcus.

\section{Introduction}

Received for publication: May 31, 2015 Accepted: September 29, 2015

Correspondence to: Helia Bello-Toledo Laboratorio de Investigación en Agentes Antibacterianos Departamento de Microbiología Facultad de Ciencias Biológicas Universidad de Concepción, Concepción, Chile Phone: +56 $412661201 \quad$ Fax: +56 412245975 E-mail: hbello@udec.cl
One of the prevalent causes of lesions that appear or remain after endodontic treatment is the presence of microorganisms or contamination in the root canal system $^{1}$. Several authors reported that bacteria could survive within the root canal even after careful mechanical-chemical preparation ${ }^{2}$. Such bacterial presence may lead to clinical symptoms of persistent chronic apical periodontitis (CAP), which has high prevalence in some countries, ranging from $40 \%$ to $61 \%^{3-4}$. The treatment of persistent CAP aims the removal of root canal filling material, disinfection of canals with irrigants and use of intra canal pastes such as calcium hydroxide. Even so, sometimes the microorganisms persist even after treatment ${ }^{5}$.

In cases of primary endodontic infections, there is a higher bacterial load than in secondary infections ${ }^{6-7}$. After biomechanical preparation of root canals, 
the bacterial load is reduced by at least $95 \%$ and the remaining viable bacteria may be the cause of refractory lesions ${ }^{8}$. Teeth with endodontic failure have been associated with the presence of Enterococcus species ${ }^{9-10}$. These bacteria probably reach the root canal system after treatment, but the source is still unclear. Enterococci do not seem to be colonizers of the oral cavity, unless there is a preferable site, such as a necrotic or obturated root canal without sealing. Probably, the transient microorganisms in the oral cavity and changes in the microenvironment create favorable conditions for secondary or refractory endodontic infection ${ }^{11}$. Enterococcus faecalis is a commensal organism, part of the normal microbiota of the digestive system, but it can also be an opportunistic human pathogen. Moreover, E. faecalis has the ability to maintain its viability for twelve months without additional nutrients. This bacteria is most frequently isolated in CAP cases after endodontic tooth treatment, but it may also be found in cases of necrotic pulps in very few cases ${ }^{12}$. Viable E. faecalis strains could be trapped inside the root canal in the filling material and provide a niche for subsequent infection in the long term ${ }^{13}$.

In Chile, there are yet no studies about the presence of this bacterial genus in CAP. The aim of this study was to identify Enterococcus spp. from the root canals of teeth that had previous endodontic treatment with diagnosis of CAP in a Chilean population and to correlate its presence with the patient's clinical features.

\section{Material and methods}

\section{Patient Selection}

Twenty adult patients, aged eighteen and older, were chosen. All were treated at the Faculty of Dentistry, University of Concepción, Chile, in November 2013, with endodontic retreatment indicated in the mandibular and maxillary canine, premolar, first or second molar. Teeth that had endodontic treatment two or more years before and showed radiographic evidence of periapical disease were considered to require retreatment. The radiolucent area size was recorded, and classified as widened periodontal ligament space $(<1 \mathrm{~mm})$ or periapical lesion $(\geq 1 \mathrm{~mm})$. Pre-treatment quality was recorded after radiographic examination. Root canal fillings with clearly visible voids in the filling material were classified as inadequate. Patients who had antibiotic treatment during the preceding three months and whose teeth could not be fully isolated with a rubber dam and/or with periodontal disease in the selected or adjacent teeth were excluded. The following characteristics for each patient and correlated with the microbial findings were recorded: age, sex, tooth type, and time when the previous treatment was performed. Clinical signs and symptoms included the quality of the dental crown restoration, spontaneous pain, pain history, sensitivity to percussion, pain on palpation, mobility, history of antibiotic therapy or any other relevant medication?

The Ethics Committee of the Faculty of Dentistry at the University of Concepción approved the study protocol (C.I.Y.B. №08/14). Before sampling, patients understood and gave written informed consent, according to the Declaration of Helsinki.

\section{Sampling Procedure}

A single trained operator collected samples under strict asepsis, as described below. The tooth surface was cleaned with pumice stone powder and isolated with a rubber dam. The tooth and the operation field were disinfected with 3 $\mathrm{mL}$ of $5.25 \%$ sodium hypochlorite solution, which was then absorbed with sterile gauze. The solution was inactivated with $5 \%$ sodium thiosulphate in order to avoid interference with the bacteriological sampling ${ }^{14}$.

Coronal restorations were removed using high-speed sterile carbide drills. To expose the root filling, the access cavity was prepared with sterile drills (Denstply, Maillefer, Switzerland) without water spray but under manual irrigation with sterile saline solution (B. Braun, Barcelona, Spain). Then, the operating field, including the pulp chamber, rubber dam and clamp, was cleaned with $2.5 \%$ sodium hypochlorite solution (Clorox Regular Bleach, Oakland, CA, USA), and inactivated with $5 \%$ sodium thiosulphate (Hash, Loveland, CO, USA). To control the effectiveness of disinfection, samples from the operating field were obtained and the tooth crown was disinfected with two F1 paper points (Denstply, Maillefer, Switzerland). Next, they were transferred to a tube containing $3 \mathrm{~mL}$ of trypticase soy broth (TSB) (Difco, Detroit, MI, USA) and incubated for $72 \mathrm{~h}$ at $35{ }^{\circ} \mathrm{C}$. The root canal filling was removed with Hedström Files, $\mathrm{K}$ and/or H type, and GatesGlidden drills (Denstply, Maillefer, Switzerland). ProTaper nickel-titanium retreatment instruments D1 - D3 (Denstply, Maillefer, Switzerland) were also used. Solvent was not used in order to avoid harm to bacterial cells. The working length was set at $1 \mathrm{~mm}$ from the radicular apex. Radiographs were taken to ensure that all the filling material had been removed. Subsequently, 5 TSB drops were placed in the root canal and a sterile endodontic file was introduced at a level of approximately $1 \mathrm{~mm}$ from the apex of the tooth. The content from the root canal was absorbed with 4 sterile paper points. Each paper point was maintained in position inside the duct at the established working length for one minute and then transferred into a tube with $1 \mathrm{~mL} \mathrm{TSB}{ }^{15}$. From this sample, an aliquot was taken to perform bacterial counts and the remainder was incubated for $24-48 \mathrm{~h}$. If the plate count was negative, the remainder of the sample was allowed to seed on a KF Streptococcus agar plate (Merck, D-61 Darmstadt, Germany) to definitively rule out the presence of Enterococcus spp. ${ }^{5,16}$.

\section{Bacterial Count}

The bacterial count was carried out in triplicate by seeding onto the surface of KF Streptococcus agar plates (Merck, D-61 Darmstadt, Germany). Plates were incubated for $72 \mathrm{~h}$ at $37{ }^{\circ} \mathrm{C}$. Subsequently, six presumptive Enterococcus spp. colonies were chosen from each patient sample.

\section{Metabolic and biochemical strain characterization}

To identify the presumptive Enterococcus spp. colonies, 
conventional tests, such as Gram staining, growth in $6.5 \%$ $\mathrm{NaCl}$ broth, esculin hydrolysis and the arabinose fermentation were carried out ${ }^{17}$. Each Enterococcus spp. strain was stored at $-80{ }^{\circ} \mathrm{C}$ in a TSB and $50 \%$ glycerol mixture at a ratio of 2:1.

\section{Molecular identification by PCR}

Identification at the species level was performed by PCR using the specific primers 5'-ATC AAG TAC AGT TAG TCT T-3' and 5'-ACG ATT CAA AGC TAA CTG-3' targeted against the $d d l$ E. faecalis gene, 5'-GCA AGG CTT CTT AGA GA-3' and 5'-CAT CGT GTA AGC TAA CTT C-3' targeted against ddl E. faecium gene, and 5'-GGT ATC AAG GAA ACC TC3' and 5'-CTT CCG CCA TCA TAG CT-3' targeted against the vanC-1 E. gallinarum gene ${ }^{18-19}$. The ADN template was obtained according to Sepulveda et al. (2002) ${ }^{18}$. Briefly, four Enterococcus spp. colonies after $24 \mathrm{~h}$ culture were suspended in $200 \mu \mathrm{L}$ sterile distilled water (SDW) and centrifuged for $5-10 \mathrm{~s}$ at $14,000 \mathrm{rpm}$. Then, $2 \mu \mathrm{L}$ of the supernatant from the suspension were added to a reaction mixture containing 1 $\mu \mathrm{L} 50 \mathrm{mM} \mathrm{MgCl}$ (Invitrogen, Carlsbad, CA, USA), $2.5 \mu \mathrm{L}$ $1.25 \mathrm{mM}$ dNTPs (Invitrogen, Carlsbad, CA, USA), $2.0 \mu \mathrm{L}$ of each primer $(20 \mathrm{pmol} / \mu \mathrm{L})$ (Integrated DNA Technologies, Coralville, IA, USA), $2.5 \mu \mathrm{L}$ of 10X PCR buffer (Invitrogen, Carlsbad, CA, USA), $0.15 \mu \mathrm{L}$ of recombinant Taq DNA polymerase $(5 \mathrm{U} / \mu \mathrm{L})$ (Invitrogen, Carlsbad, CA, USA) and adequate SDW to complete a $25 \mu \mathrm{L}$ reaction volume. Escherichia coli ATCC 25922, E. faecalis ATCC 29212, E. faecium VRE 11, and E. gallinarum 132674 strains, available at the Culture Collection Department of Microbiology at the University of Concepción, were used as controls in PCR. The amplification was performed in a Veriti 96-well thermal cycler (Applied Biosystems, Foster City, CA, USA) under the following program: $2 \mathrm{~min}$ at $94{ }^{\circ} \mathrm{C}$ and 30 cycles at 94 ${ }^{\circ} \mathrm{C}, 1 \mathrm{~min}$ at $54{ }^{\circ} \mathrm{C}$ and $1 \mathrm{~min}$ at $72{ }^{\circ} \mathrm{C}$, with a final step of 10 min at $72{ }^{\circ} \mathrm{C}$. Amplicons were separated by electrophoresis in $1.5 \%$ agarose gel at $100 \mathrm{~V}$ for $30-45 \mathrm{~min}$ in $0.5 \mathrm{X} \mathrm{TAE}$ buffer and stained by ethidium bromide $(0.5 \mu \mathrm{g} / \mathrm{mL})$ for 15 min, followed by washing in SDW for $15 \mathrm{~min}$. DNA bands were visualized with a UV White Light transilluminator (Vilber Lourmat, Torcy, Z.I. Sud, France) ${ }^{15,18}$. Later, gels were photographed with a Kodak ID LPU 120 camera (Rochester, NY, USA) and analysed by the ID 3.02 Kodak Digital Science Software (Rochester, NY, USA).

\section{Data Analysis}

Prevalence of Enterococcus spp. was recorded as percentage of positive cases. The relationship between the presence of $E$. faecalis and the clinical variables was analysed with the Pearson, chi-square and Fisher's exact tests using SPSS version 15 (SPSS Inc., Chicago, IL, USA). The results of PCR assay to detect $E$. faecalis in clinical samples were analysed with the proportion comparison z-test, adopting a 0.05 significance level.

\section{Results}

Presumptive colonies of enterococci were found on KF
Streptococcus agar plate in 14 out of 20 samples obtained from the root canals. The colonies were circular with convex elevation and smooth margins, deep red and $1 \mathrm{~mm}$ diameter (Figure 1). The bacterial counts ranged from zero CFU $/ \mathrm{mL}$ to $2.1 \times 10^{5} \mathrm{CFU} / \mathrm{mL}$. They represent the distribution of $E$. faecalis countings over the samples (Table 1). After regrowth time, aliquots seeded again in KF agar, reporting some colonies (few) not characteristic to Enterococcus, presumably positive for other microorganism.

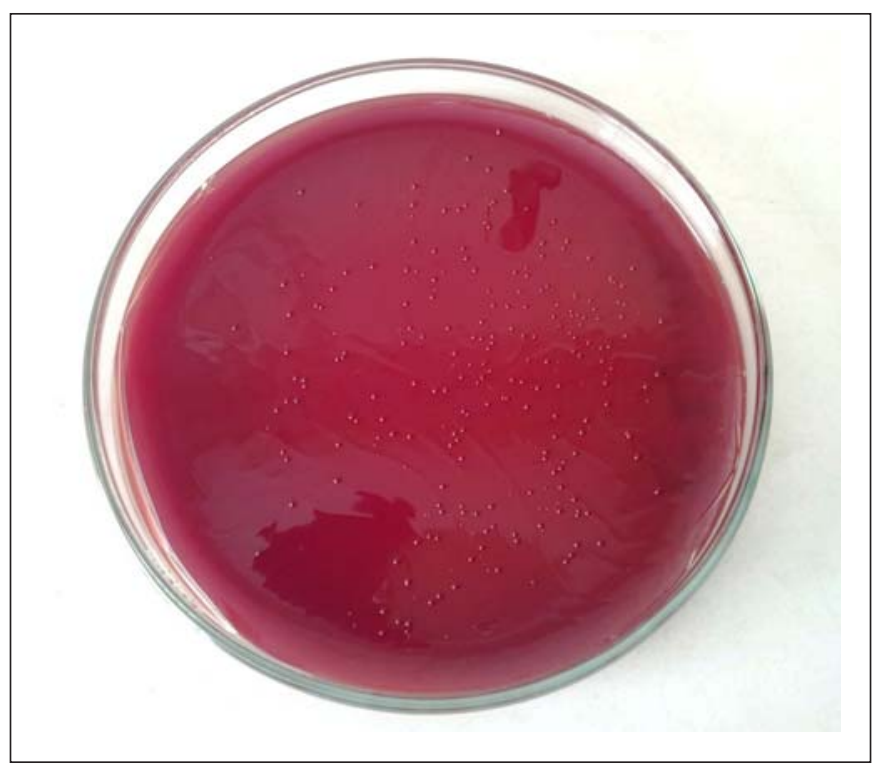

Fig. 1: Enterococcus spp. colony forming units (CFUs) on KF agar plate $\left(10^{-3}\right.$ dilution).

Table 1: Counts of E. faecalis from root canals with persistent chronic apical periodontitis

\begin{tabular}{lccc}
\hline Sample & $\begin{array}{c}\text { Median } \\
\text { (CFU/mL) }\end{array}$ & $\begin{array}{c}\text { Minimum } \\
\text { (CFU/mL) }\end{array}$ & $\begin{array}{c}\text { Maximum } \\
\text { (CFU/mL) }\end{array}$ \\
1 & 0 & 0 & 0 \\
2 & $2 \times 10^{1}$ & 0 & 9 \\
3 & 9 & 0 & 9 \\
4 & $1.3 \times 10^{3}$ & $1.3 \times 10^{2}$ & $1.5 \times 10^{3}$ \\
5 & $1.4 \times 10^{3}$ & $1.5 \times 10^{2}$ & $1.4 \times 10^{3}$ \\
6 & 9 & 0 & 9 \\
7 & $1.1 \times 10^{3}$ & $9 \times 10^{1}$ & $1.3 \times 10^{3}$ \\
8 & $3.4 \times 10^{3}$ & $3.5 \times 10^{2}$ & $7 \times 10^{3}$ \\
9 & $8.5 \times 10^{4}$ & $7.9 \times 10^{4}$ & $9.1 \times 10^{4}$ \\
10 & $4 \times 10^{1}$ & 0 & $4 \times 10^{1}$ \\
11 & 0 & 0 & 0 \\
12 & $2.1 \times 10^{5}$ & $2 \times 10^{5}$ & $2.1 \times 10^{5}$ \\
13 & 0 & 0 & 0 \\
14 & 0 & 0 & 0 \\
15 & 9 & 0 & 9 \\
16 & 9 & 0 & 9 \\
17 & 0 & 0 & 0 \\
18 & $8 \times 10^{1}$ & $5 \times 10^{1}$ & $10 \times 10^{1}$ \\
19 & 0 & 0 & 0 \\
20 & 9 & 0 & 9 \\
\hline
\end{tabular}

$\mathrm{CFU}=$ Colony Forming Unit 
Phenotypic identification indicated that $83 / 84$ (98\%) selected strains corresponded to E. faecalis, which was confirmed by PCR, as amplified bands of $941 \mathrm{bp}$ were obtained with the specific primers for this species in all assays (Figure 2). E. faecium (2\%) was the only strain with different characteristics identified through phenotypic and molecular tests. In this case, a 550 bp amplification product was obtained using specific primers for this species (Figure 3).

Regarding the clinical aspects, no significant differences were found between gender, age and medical history variables. Most teeth with presence of Enterococcus spp. were maxillary and posterior. For the variable related to time after previous endodontic treatment, increased presence of Enterococcus spp. was observed in the range of two to five years. For the variable regarding quality of root canal filling and dental crown restorations, a high microorganism frequency was found in treatments labelled as "inadequate". In relation to the clinical evaluation, there was a direct association between the absence of signs and symptoms and the presence of Enterococcus spp. For the periapical variable, there was no association between the lesion size and the count. However, no statistically significant relationship was found between the clinical variables and bacterial count.

\section{Discussion}

Due to the physical limitations of the root canal system, obtaining a representative sample from this site is not an easy task, and it is even more difficult in patients with an indication for retreatment, in which the number of microorganisms may be low and a significant number of microbial cells can be lost during root canal desobturation procedures. As a result, the number of bacterial cells in the sample can fall below the detection rate of the isolation method and a particular species' prevalence may be underestimated $^{20}$. This was one limitation of the present study, in which the impossibility of using liquid solvents made the surgical procedure last for three hours in order to completely

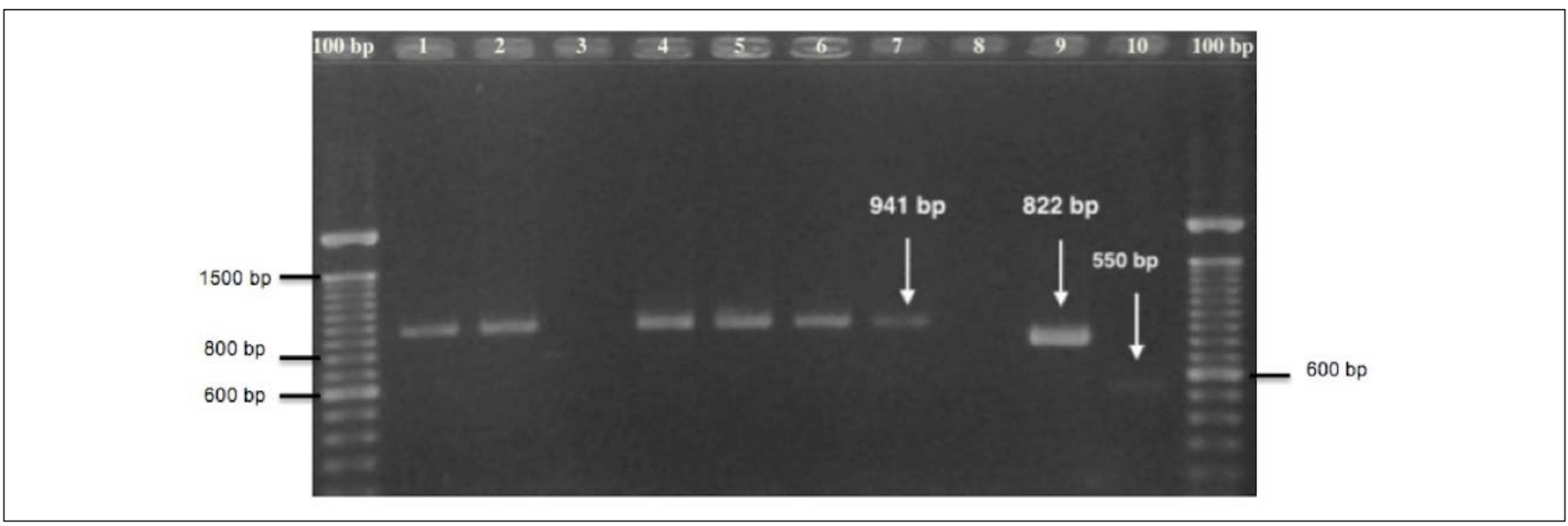

Fig. 2: Identification by PCR of Enterococcus spp. isolated from patient 6. Lane 1 to 6: six strains obtained from patient 6. Lane 7: positive control (E. faecalis ATCC 29212). Lane 8: negative control: E. coli ATCC 25922. Lane 9: positive control (E. gallinarum 132674). Lane 10: positive control (E. faecium VRE 11).

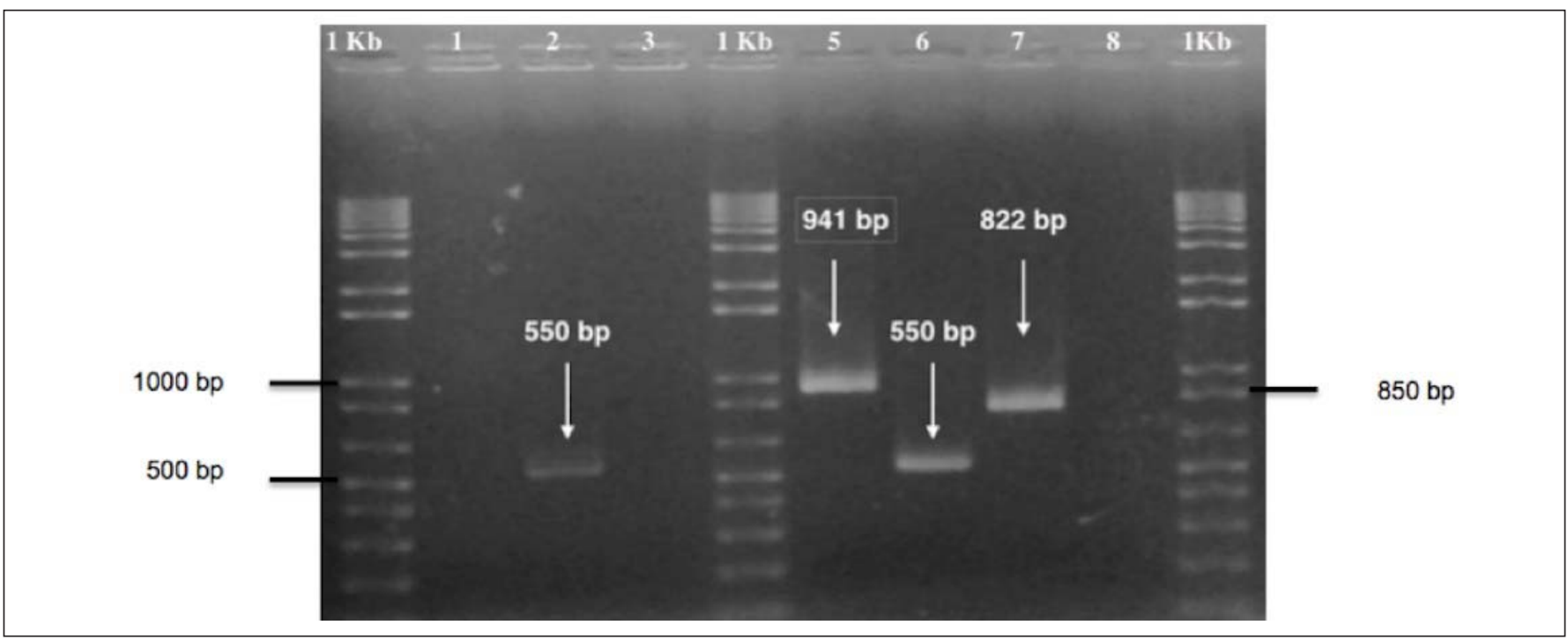

Fig. 3: Identification by PCR of $E$. faecium isolated from patient 6 . Lane 1: patient 6 strain 3 with primers for $E$. faecalis. Lane 2: patient 6 strain 3 with primers for $E$. faecium. Lane 3: patient 6 strain 3 with primers for E. gallinarum. Lane 5: positive control (E. faecalis ATCC 29212). Lane 6: positive control (E. faecium VRE 11). Lane 7: positive control (E. gallinarum 132674). 
remove all root canal filling material. This could have influenced those cases that were negative for enterococci. The appearance of some colonies (few) not characteristic to Enterococcus can be explained because in the $\mathrm{KF}$ Streptococcus agar may grow other species like Listeria monocytogenes, Streptococcus bovis group, Pediococcus and staphylococci. Other organisms (e.g., micrococci, Candida, corynebacteria and gram-negative bacteria) may appear as clear small colonies or produce growth traces.

A study carried out to compare the detection rate of $E$. faecalis from cultivation and PCR test from the same sample found that $46.6 \%$ of the sampled canals had E. faecalis ${ }^{14}$. In a Lithuanian population study, $56 \%$ of the cases were reported to be positive for $E$. faecalis, using only culture techniques for identification ${ }^{21}$. In this study, $45 \%$ of the samples were positive for Enterococcus spp. using culture techniques. However, performing sample regrowth and subsequent seeding again on KF Streptococcus agar plates, the detection percentage yielded up to $70 \%$, depending on the total number of samples, which was confirmed by the PCR test; which is consistent with previous studies. It should be underscored that the sample size in the present case is smaller than in other studies and it may have influenced the higher percentage obtained. The authors know that sample size calculation may determine the exact number of samples is required to strictly determine the prevalence of Enterococcus spp. The authors developed a descriptive, cross-sectional study. Relative frequency Enterococcus spp isolated from root canals was evaluated in a limited time. In this case, the sample was not probabilistic. It was taken depending on availability of filed cases in a given time. This limited the number of sampled patients.

The prevalence of $E$. faecalis and its association with clinical findings among patients in Beijing, China, showed that root canal treatments displaying unsatisfactory obturation presented a greater diversity of cultivable species than those with satisfactory obturation ${ }^{22}$. Those canals probably provided more space and nutrition than wellobturated canals. In fact, in that study, E. faecalis was more frequent in the canals than in saliva, but this was not the aim of our study. On the contrary, the well-obturated canals maintained an obliged anaerobic environment, which does not favor the survival and growth of E. faecalis. In that Chinese study, as well as in the present study, no association was found between these clinical features and the prevalence of E. faecalis in radicular canals, which is also attributed to the low sensitivity of Fisher's exact test in small samples ${ }^{22}$. $E$. faecalis has been commonly found in filled canals with radiographic evidence of peri-radicular periodontitis or without injury than in those with a radiolucent area, suggesting that the entry of bacteria may occur after filling, which is consistent with the findings of this study ${ }^{23}$. The observation of similar percentages of E. faecalis leakage, regardless of the obturation technique, reinforces this idea ${ }^{24}$.

Confirmation of the identity of $E$. faecalis by PCR has proven to be more sensitive than conventional $E$. faecalis culture methods. In this study, the use of a selective medium (KF Streptococcus agar) and some biochemical tests enabled us to focus on the identification of this bacterium in relation to other species. In this case, there was no presence of $E$. faecalis in $30 \%$ of the samples in comparison with other reports of $6.8 \%{ }^{25}$ to $20 \%{ }^{21}$ of teeth. Combining molecular and culture techniques, including biochemical profile is most likely the best available method to provide a comprehensive information on the bacterial populations associated with endodontic infections. Ultimately, the observation that $98.8 \%$ of the strains were $E$. faecalis versus the $1.2 \%$ that turned out to be $E$. faecium was a probable outcome, considering the results of a study on non-dental clinical isolates from a hospital environment in a Chilean population ${ }^{18}$. It should be stressed that the most relevant clinical characteristic of one of the patients, from whom the strain of E. faecium was isolated, was that he presented the worst quality of obturation, which was evident in the root canal filling material, the absence of dental crown restorations and sensitivity to palpation. However, his initial count was $<10 \mathrm{CFU} / \mathrm{mL}$ as an estimated value.

Clearly, further studies are required to analyse other enterococci populations that may be linked to the presence and establishment of refractory chronic apical periodontitis during or after endodontic treatment, and which may form a multi-species biofilm. E. faecalis has been regarded for more than 30 years as a leading cause of endodontic failures. However, this assertion was placed in doubt based on recent publications reporting that other species, such as streptococci, may be predominant pathogens associated with persistent endodontic infections. The difference may be due to different methodological approaches for sampling and detection, different clinical conditions or socio-geographical differences in the studied subjects ${ }^{6,18,26}$. Finally, it is necessary to advocate for a comprehensive approach to find new antibacterial substances that completely eradicate the microorganisms from the root canal system, which in its genesis is formed to maintain sterility ${ }^{27}$.

This study has shown that there is a high prevalence of $E$. faecalis in root canal-treated teeth in a Chilean population. Further studies are required to analyse the presence and role of other species, which may be linked to refractory chronic apical periodontitis.

\section{Acknowledgements}

The authors wish to acknowledge the support of the Faculty of Dentistry of the University of Concepción, the Public Health Institute of Chile for supplying the strain of $E$. faecium VRE 11 ( $\operatorname{van} B$ ) and the Research Laboratory on Antibacterial Agents of the Faculty of Biological Sciences at the University of Concepción. This study was supported by the Scholarship "CONICYT PCHA/National Doctorate/ 2013-folio 21130022", for PhD in Sciences, Mention in Microbiology, University of Concepción, Chile.

\section{References}

1. Ricucci D, Siqueira JF Jr. Anatomic and microbiologic challenges to achieving success with endodontic treatment: a case report. J Endod. 2008; 34: 1249-54. 
2. Rasimick B, Shah R, Musikant B, Deutsch A. Bacterial colonization of root canal dentine previously treated with endodontic irrigants. Aust Endod J. 2010; 36: 70-3.

3. Tsesis I, Goldberger T, Taschieri S, Seifan M, Tamse A, Rosen E. The dynamics of periapical lesions in endodontically treated teeth that are left without intervention: a longitudinal study. J Endod. 2013; 39: 1510-5.

4. Mukhaimer R, Hussein E, Orafi I. Prevalence of apical periodontitis and quality of root canal treatment in an adult Palestinian sub-population. Saudi Dent J. 2012; 24: 149-55.

5. Pavaskar R, de Ataide ID, Chalakkal P, Pinto MJ, Fernandes KS, Keny $\mathrm{RV}$ et al. An in vitro study comparing the intra canal effectiveness of calcium hydroxide and linezolid-based medicaments against Enterococcus faecalis. J Endod. 2012; 38: 95-100.

6. Hong BY, Lee TK, Lim SM, Chang SW, Park J, Han SH et al. Microbial analysis in primary and persistent endodontic infections by using pyrosequencing. J Endod. 2013; 39: 1136-40.

7. Ozok AR, Persoon IF, Huse SM, Keijser AR, Wesselink PR, Crielaard $W$ et al. Ecology of the microbiome of the infected root canal system: a comparison between apical and coronal root segments. Int Endod J. 2012; 45: 530-41.

8. Blome B, Braun A, Sobarzo V, Jepsen S. Molecular identification and quantification of bacteria from endodontic infections using real-time polymerase chain reaction. Oral Microbiol Immunol. 2008; 23: 384-90.

9. Gomes BP, Pinheiro E, Sousa EL, Jacinto RC, Zaia AA, Randi CC et al. Enterococcus faecalis in dental root canals detected by culture and by polymerase chain reaction analysis. Oral Surg Oral Med Oral Pathol Oral Radiol Endod. 2006; 102: 247-53.

10. Figdor $D$, Sundqvist $G$. A big role for the very small - understanding the endodontic microbial flora. Aust Dental J Suppl. 2007; 52: S38-51.

11. Zehnder M, Guggenheim B. The mysterious appearance of enterococci in filled root canals. Int Endod J. 2009; 42: 277-87.

12. Sedgley CM, Buck G, Appelbe O. Prevalence of Enterococcus faecalis at multiple oral sites in endodontic patients using culture and PCR. J Endod. 2006; 32: 104-9.

13. Vidana $\mathrm{R}$, Sullivan $\mathrm{A}$, Billstrom $\mathrm{H}$, Ahlquist M, Lund B. Enterococcus faecalis infection in root canals - host-derived or exogenous source? Lett Appl Microbiol. 2011; 52: 109-15.

14. Gomes BP, Pinheiro ET, Jacinto RC, Zaia AA, Ferraz CC, de SouzaFilho FJ. Microbial analysis of canals of root-filled teeth with periapical lesions using polymerase chain reaction. J Endod. 2008; 34: 537-40.

15. Zoletti GO, Siqueira JF, Santos KR. Identification of Enterococcus faecalis in root-filled teeth with or without periradicular lesions by culture-dependent and-independent approaches. J Endod. 2006; 32: 722-6.

16. Hoben HJ, Somasegaran P. Comparison of the Pour, Spread, and Drop Plate Methods for Enumeration of Rhizobium spp. in Inoculants Made from Presterilized Peat. Appl Environ Microbiol. 1982; 44: 1246-7.

17. Facklam RR, Collins MD. Identification of Enterococcus species isolated from human Infections by a conventional test scheme. J Clin Microbiol. 1989; $27: 731-4$

18. Sepulveda M, Bello H, Ruiz M, Hormazábal F, Dominguez M, González $G$ et al. Classic and molecular methodologies for the identification of Enterococcus species. Rev Med Chile. 2002; 130: 45-9.

19. Dutka-Malen S, Evers S, Courvalin P. Detection of glycopeptide resistance genotypes and identification to the species level of clinically relevant enterococci by PCR. J Clin Microbiol. 1995; 33: 24-7.

20. Rocas IN, Siqueira JFJ. Characterization of microbiota of root canal-treated teeth with posttreatment disease. J Clin Microbiol. 2012; 50: 1721-4.

21. Peciuliene V, Balciuniene I, Eriksen HM, Haapasalo M. Isolation of Enterococcus faecalis in previously root-filled canals in a Lithuanian population. J Endod. 2000; 26: 593-5.

22. Wang QQ, Zhang CF, Chu CH, Zhu XF. Prevalence of Enterococcus faecalis in saliva and filled root canals of teeth associated with apical periodontitis. Int J Oral Sci. 2012; 4: 19-23.
23. Kaufman B, Spångberg L, Barry J, Fouad AF. Enterococcus spp. in endodontically treated teeth with and without periradicular lesions. J. Endod. 2005; 31: 851-6.

24. Nabeshima CK, Rosa Martins GH, Pasquali Leonardo MF, Furukava Shin, Silvana Cai RC, Lima Machado ME. Comparison of three obturation techniques with regard to bacterial leakage. Braz J Oral Sci. 2013; 12: 212-5.

25. Zhu X, Wang Q, Zhang C, Cheung GS, Shen Y. Prevalence, phenotype, and genotype of Enterococcus faecalis isolated from saliva and root canals in patients with persistent apical periodontitis. J Endod. 2010; 36: 1950-5.

26. Tennert C, Fuhrmann M, Wittmer A. Karygianni L, Altenburger M, Pelz K et al. New Bacterial Composition in Primary and Persistent/ Secondary Endodontic Infections with Respect ( to Clinical and Radiographic Findings J Endod. 2014; 40: 670-7.

27. Paloma B, Cabral dos Santos C, Alves F, Lima L, Machado C. In Vitro antimicrobial photoinactivation with methylene blue in different microorganisms. Braz J Oral Sci. 2014; 13: 53-7. 